\title{
Beam test of a new radio frequency quadrupole linac for the Japan Proton Accelerator Research Complex
}

\author{
Yasuhiro Kondo," Takatoshi Morishita, Saisyun Yamazaki, Toshihiko Hori, Yuki Sawabe, \\ Etsuji Chishiro, Shinpei Fukuta, Kazuo Hasegawa, Koichiro Hirano, Nobuhiro Kikuzawa, \\ Isao Koizumi, Akihiko Miura, Hidetomo Oguri, Kiyonori Ohkoshi, Fumiaki Sato, \\ Shinichi Shinozaki, and Akira Ueno \\ Japan Atomic Energy Agency (JAEA), Tokai, Naka, Ibaraki 319-1195, Japan
}

Hiroshi Kawamata, Takashi Sugimura, Akira Takagi, Zhigao Fang, Yuji Fukui, Kenta Futatsukawa, Kiyoshi Ikegami, Tomofumi Maruta,

Tomoaki Miyao, and Kesao Nanmo

High Energy Accelerator Research Organization (KEK), Tsukuba, Ibaraki 305-0801, Japan (Received 22 August 2014; published 19 December 2014)

\begin{abstract}
We performed a beam test of a new radio frequency quadrupole linac (RFQ III) for the beam current upgrade of the Japan Proton Accelerator Research Complex. First, the conditioning of RFQ III was conducted, and after $20 \mathrm{~h}$ of conditioning, RFQ III became very stable with a nominal peak power and duty factor of $400 \mathrm{~kW}$ and $1.5 \%$, respectively. An off-line beam test was subsequently conducted before installation in the accelerator tunnel. The transmission, transverse emittance, and energy spread of the 50-mA negative hydrogen beam from RFQ III were measured and compared with simulation results. The experiment and simulation results showed good agreement; therefore, we conclude that the performance of RFQ III conforms to its design.
\end{abstract}

DOI: 10.1103/PhysRevSTAB.17.120101

PACS numbers: 29.20.Ej, 29.27.Fh

\section{INTRODUCTION}

The Japan Proton Accelerator Research Complex (J-PARC) is a multipurpose facility for particle physics, nuclear physics, materials and life science, and other applications. The J-PARC accelerator [1] consists of a $400-\mathrm{MeV}$ linac, a 3-GeV rapid cycling synchrotron, and a $50-\mathrm{GeV}$ main ring. The original design energy and peak beam current of the linac are $400 \mathrm{MeV}$ and $50 \mathrm{~mA}$, respectively. A four-vane-type radio frequency quadrupole (RFQ) built for the Japan Hadron Facility (JHF) project was used for the initial phase linac, and the design peak beam current of this RFQ was $30 \mathrm{~mA}$ [2]. To upgrade the beam current of the J-PARC linac to achieve the original design power of $1 \mathrm{MW}$ (at the neutron target), a new RFQ with a design current of $50 \mathrm{~mA}$ has been developed [3]. This RFQ is called RFQ III, whereas the former 30-mA RFQ is called RFQ I (a brief history of the J-PARC RFQs is described in Sec. II). Table I lists the design parameters of RFQ III.

RFQ III employs a conventional beam dynamics design. That is, the RFQ is divided into four sections: a radial matching section (RMS), a shaper (SP), a gentle buncher (GB), and an accelerator (ACC). Additionally, the intervane

\footnotetext{
*yasuhiro.kondo@j-parc.jp
}

Published by the American Physical Society under the terms of the Creative Commons Attribution 3.0 License. Further distribution of this work must maintain attribution to the author $(s)$ and the published article's title, journal citation, and DOI. voltage $V$ and the average bore radius $r_{0}$ are maintained constant except for the injection section; this means that the focusing strength $B$ is maintained constant. The merits of this design are as follows. First, if $r_{0}$ and $V$ are constant, the resonant frequency of the cross-sectional shape can be constant, and therefore, frequency tuning using the vaneskirt shape or cavity radius is not required. Thus, the longitudinally uniform cross-sectional shape affords easy design and machining of the cavity. Second, it is possible to machine constant- $r_{0}$ and constant- $\rho_{t}$ ratio vanes using formed cutters. Even if ball nose cutters are used, the computer-aided machining program for constant-crosssection vanes is very simple. Moreover, the field distribution is uniform along the RFQ, and this makes it easier to tune the cavity in the fabrication process. Additionally, the stability during operation is expected to be better because of the longitudinally uniform structure. Considering these merits and our experience with RFQ I and RFQ II, the conventional constant- $r_{0}$ and constant $-V$ design was adopted for J-PARC RFQ III.

For the beam dynamics design of RFQ III, LINACSRFQDES [4,5] was used [3]. The feature that characterizes the beam dynamics in LINACSRFQDES is the equipartitioning condition, which is used to avoid the effect of parametric resonances in high current linacs [6]. This condition requires that the internal energies in the transverse and longitudinal phase spaces of the beam are the same, i.e., 
TABLE I. Design parameters of J-PARC RFQ III.

\begin{tabular}{ll}
\hline \hline Beam species & $\mathrm{H}^{-}$ \\
Resonant frequency & $324 \mathrm{MHz}$ \\
Injection energy & $50 \mathrm{keV}$ \\
Extraction energy & $3 \mathrm{MeV}$ \\
Peak beam current & $50 \mathrm{~mA}$ \\
Vane length & $3623 \mathrm{~mm}$ \\
Average bore radius $\left(r_{0}\right)$ & $3.49 \mathrm{~mm}$ \\
$\rho_{t} / r_{0}$ ratio ${ }^{\mathrm{a}}$ & $0.75\left(\rho_{t}=2.62 \mathrm{~mm}\right)$ \\
Intervane voltage & $81.0 \mathrm{kV}$ \\
Maximum surface field & $30.7 \mathrm{MV} / \mathrm{m}(1.72$ Kilpatrick) \\
Nominal peak power & $400 \mathrm{~kW}$ \\
Repetition rate & $50 \mathrm{~Hz}$ \\
rf pulse length & $600 \mu \mathrm{s}$ \\
Duty factor & $3 \%$ \\
\hline \hline
\end{tabular}

${ }^{\mathrm{a}} \rho_{t}$ : transverse radius of the vane tip.

${ }^{\mathrm{b}}$ The current requirement is $25 \mathrm{~Hz}$. The $50 \mathrm{~Hz}$ operation will be required for the future upgrade of the J-PARC linac.

$$
\frac{\varepsilon_{l n} \sigma_{l}}{\varepsilon_{t n} \sigma_{t}}=1
$$

where $\varepsilon_{l n}$ and $\varepsilon_{t n}$ are the longitudinal and transverse normalized rms emittances, respectively, and $\sigma_{l}$ and $\sigma_{t}$ are the longitudinal and transverse phase advances, respectively. When this condition is satisfied, there is no free energy to drive a resonance. However, the equipartitioning condition is not maintained with maintaining $V$ constant as the particles are accelerated. Therefore, for RFQ III, we adopted equipartitioning in the GB, which is the most critical section of RFQs.

In accordance with the concepts described above, J-PARC RFQ III was designed and fabricated [7]. Because the J-PARC linac provides a beam for user operation, the performance of RFQ III had to be confirmed by off-line testing using a negative hydrogen $\left(\mathrm{H}^{-}\right)$beam prior to replacing RFQ I. In this paper, the beam test results of J-PARC RFQ III are described.

First of all, the history of the J-PARC RFQs is briefly summarized in Sec. II. In Sec. III, the experimental apparatus is presented, and the conditioning results of the RFQ are given in Sec. IV. The beam test results are presented and compared with the simulation results in Sec. V, and some points are discussed in Sec. VI. Finally, in Sec. VII, we present our conclusions.

\section{BRIEF HISTORY OF THE J-PARC RFQS}

In this section, the history of the J-PARC RFQs is summarized.

The commissioning of the J-PARC linac started in 2006, using RFQ I as a front-end accelerator. As mentioned in the previous section, this RFQ was developed for JHP, and the design peak beam current was $30 \mathrm{~mA}$. With this current, the required power of $1 \mathrm{MW}$ at the neutron target cannot be achieved, but it was our plan that we start the operation of the J-PARC accelerator complex with this RFQ and later develop a new $50 \mathrm{~mA}$ RFQ to reach the required power of $1 \mathrm{MW}$.

However, just before the start of the user operation, a sparking problem occurred in RFQ I [8]. Thus, in parallel with taking measure for the sparking of RFQ I, we decided to make a spare RFQ. We call this spare one RFQ II. Because, at that time, it was not clear RFQ I could be recovered or not, RFQ II had to be made as soon as possible. Therefore, there was no time for a new optics design, so the design of RFQ II is the same as that of RFQ I, including the peak current of $30 \mathrm{~mA}$. The high-power testing of RFQ II was successfully finished [9], but we also succeeded in reducing the trip rate of RFQ I to an acceptable level, so we did not replace RFQ I with RFQ II. Before the summer shutdown of 2014, this is the last operating period for RFQ I, RFQ I stably provided $25 \mathrm{~mA}$ beam for user operation, and had achieved $30 \mathrm{~mA}$ for accelerator study.

The development of a new 50 mA RFQ started in 2011. This is RFQ III, described in this paper.

\section{EXPERIMENTAL APPARATUS}

As mentioned in Sec. I, RFQ III was tested off-line prior to installation in the accelerator tunnel from December 2013 to June 2014. To this end, an RFQ test stand was constructed on the ground floor of the J-PARC linac building. This test stand allowed the use of a $50 \mathrm{~mA}$ beam with a duty factor of up to $25 \%$ of the nominal duty factor $(500 \mu \mathrm{s}, 25 \mathrm{~Hz})$ for continuous operation, wherein the duty factor was limited by allowable radiation levels. Figure 1 shows a schematic drawing of the test stand.

An rf driven ion source (RFIS) for the J-PARC linac upgrade [10] was employed for the RFQ testing. The plasma is driven by a pulsed $2-\mathrm{MHz}$ rf power, and a $30-\mathrm{MHz}$ continuous wave $\mathrm{rf}$ is also used to ignite the plasma. A $60-\mathrm{kW}$ solid-state amplifier system [11] is used as the $\mathrm{rf}$ source. The typical $\mathrm{rf}$ power used under the standard 60-mA beam operation was $45 \mathrm{~kW}$. The extraction energy is $50 \mathrm{keV}$. The ion source is pumped by two $1500 \mathrm{~L} / \mathrm{s}$ turbo molecular pumps (TMPs). The typical pressure under $60-\mathrm{mA}$ beam operation was $6.0 \times 10^{-3} \mathrm{~Pa}$.

The low energy beam transport (LEBT) is equipped with two solenoid magnets (SM1 and SM2), and the space charge neutralization effect is also used to focus the beam. The beam current injected to the RFQ is measured using a movable Faraday cup (FC) or a slow current transformer (SCT1) located between the two SMs. The length of the LEBT is $580 \mathrm{~mm}$. A 1500-L/s TMP, two 500-L/s TMPs, and a $2400-\mathrm{L} / \mathrm{s}$ (for $\mathrm{N}_{2}$ ) cryopump (CP) are attached to the vacuum chamber between the two SMs. Under 60-mA beam operation, the typical internal pressure was $2.0 \times 10^{-5} \mathrm{~Pa}$.

Figure 2 shows a photograph of J-PARC RFQ III. RFQ III consists of three longitudinally segmented unit cavities, and the vane lengths of the units are 1211.0, 1226.0, and 


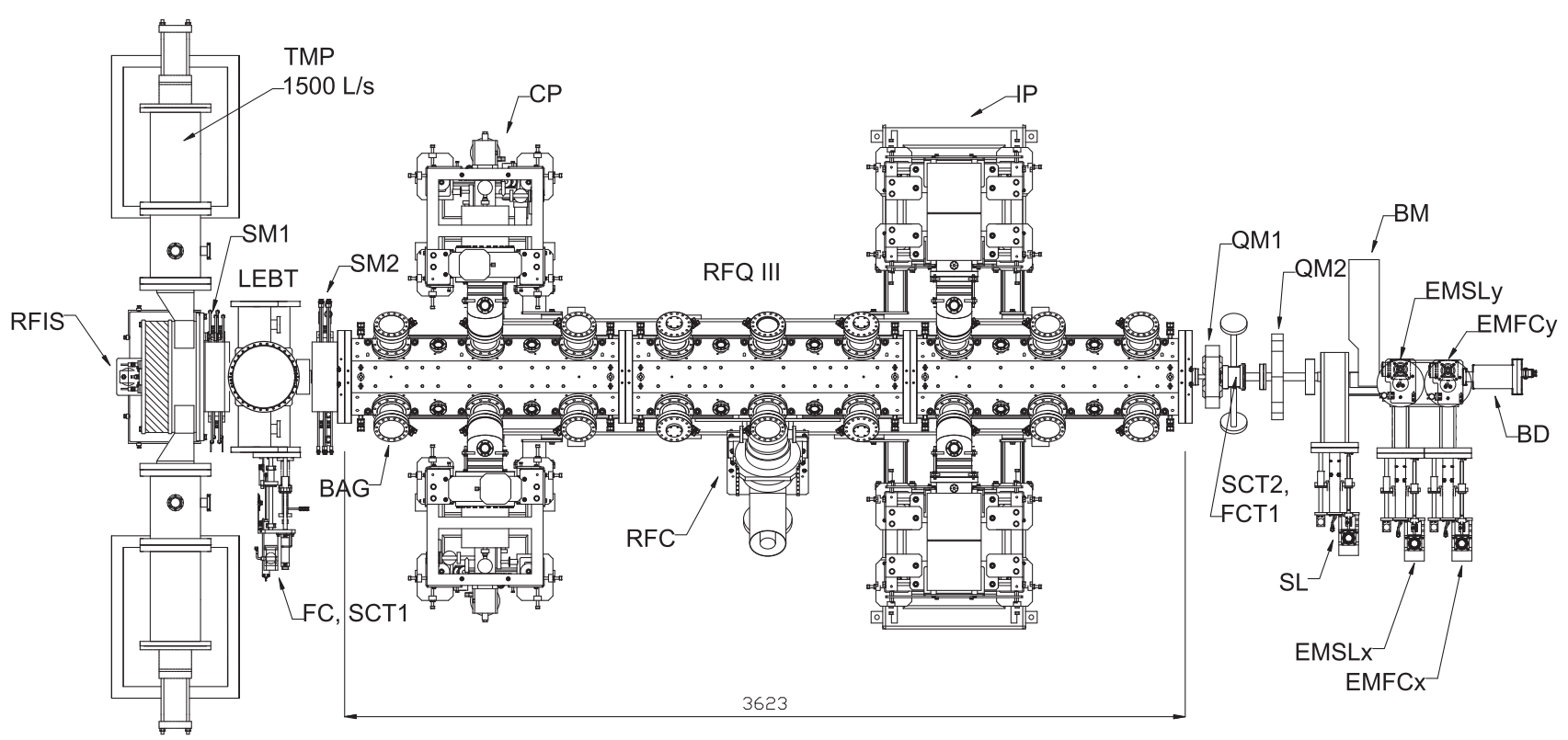

FIG. 1. Schematic of the RFQ test stand (top view).

$1186.1 \mathrm{~mm}$, respectively. Each unit cavity consists of upper and lower major vanes and left and right minor vanes. The major and minor vanes were joined by vacuum brazing. The material of the cavity is oxygen free copper class 1 [12], and hot isostatic pressing (HIP) treatment was applied to reduce the voids between the crystal grains [13]. Three 1700-L/s (for $\mathrm{N}_{2}$ ) cryopumps are attached to the second ports from the upstream direction, and four $400-\mathrm{L} / \mathrm{s}$ ion pumps (IPs) are attached to the eighth ports from the upstream direction. The pressure is measured with a Bayard-Alpert gauge attached to the most upstream port. Typical pressures were $6.0 \times 10^{-6} \mathrm{~Pa}$ with the rf off, $1.0 \times 10^{-5} \mathrm{~Pa}$ with the $\mathrm{rf}$ on, and $1.7 \times 10^{-5} \mathrm{~Pa}$ under 50-mA beam operation.

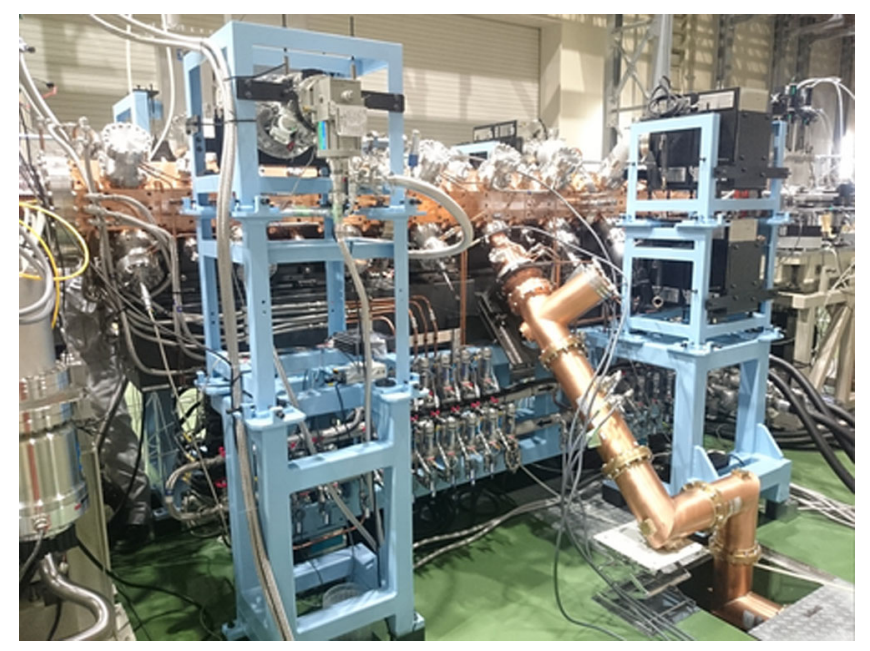

FIG. 2. Photograph of J-PARC RFQ III installed in the test stand.
In the center port of the lower-left quadrant, a loop-type rf coupler (RFC) is inserted. The rf power was generated by a 324-MHz klystron (Toshiba E3740A [14]). The rf power was transmitted via WR2300 rectangular waveguides to the vicinity of RFQ III, transformed to WX203D coaxial waveguides, reduced to $152 \mathrm{D}$, and finally reduced to $77 \mathrm{D}$ by the rf coupler.

During operation, the resonant frequency of RFQ III is tuned by controlling the cooling water temperature [9]. To this end, RFQ III is equipped with two types of cooling water channels: four channels are used to cool the vanes, and the other eight channels are used to cool the cavity walls. The diameter of the vane channels is $15 \mathrm{~mm}$ and that of the wall channels is $10 \mathrm{~mm}$. The water flow in each channel runs parallel from the low-energy end to the highenergy end, and the channels of three unit cavities are serially connected. The water channels of the three fixed tuners are connected serially, and the end plates and rf coupler each have a water channel. The water temperatures at the exits of all the channels are measured with resistance thermometers (PT100), and the flow rates are measured with float-type flow meters.

The property of the extracted beam from the RFQ was measured using a test bench. Figure 3 is a photograph of the test bench.

The beam extracted from the RFQ was transported to the beam dump (BD) using two quadrupole magnets (QM1 and QM2). The beam energy was measured to be $3.00 \mathrm{MeV}$ with the time of flight method using two fast current transformers (FCT1 and FCT2), separated by a distance of $1230 \mathrm{~mm}$. FCT2 was located just upstream of the beam dump; however, it was removed after the beam energy was confirmed because it was a cause of neutron radiation due to beam loss. 


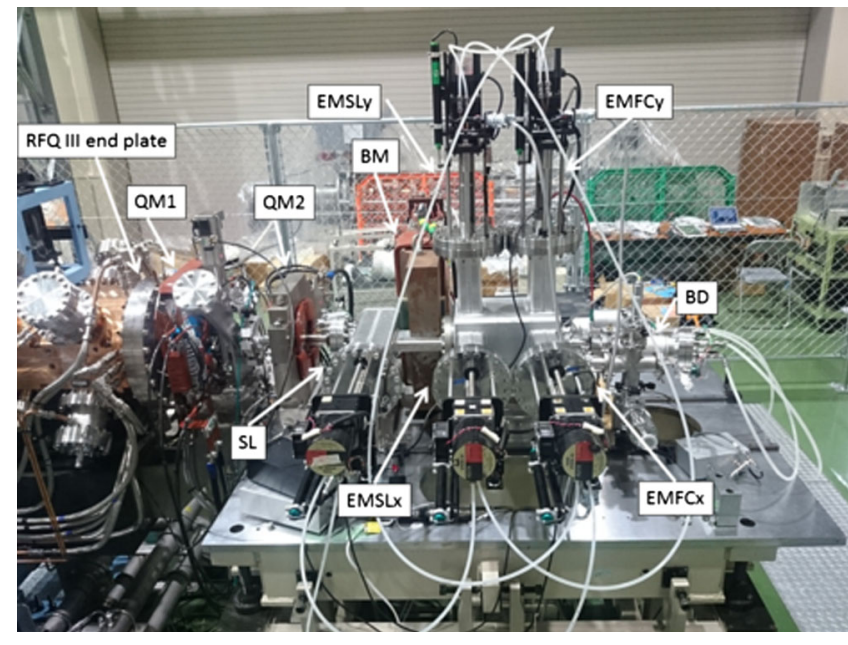

FIG. 3. Photograph of the test bench.

The energy spread was measured with an analyzer using a bending magnet (BM). The pole length of the BM was $90 \mathrm{~mm}$. The bending angle of the $3-\mathrm{MeV}$ beam was 15 degrees for a BM current of 84A. To reduce the effect of transverse emittance, a movable slit (SL) was inserted just upstream of the BM. The SL consisted of two tungsten slits, each with a gap width of $0.1 \mathrm{~mm}$. A water-cooled 8-mm copper plate was inserted between the two slits to remove the heat load on the slit. The beam profile after the BM was measured with the horizontal Faraday cup with a slit (EMFCx) of the emittance monitor.

The emittance monitor was a conventional double-slit type to measure the transverse emittances of the RFQ. The slit of the emittance monitor was composed of tungsten, which was water cooled via a copper baking plate. The gap length of the slit was $0.1 \mathrm{~mm}$, and the distance between the upstream slit (EMSL) and the downstream EMFC was $215 \mathrm{~mm}$. The charges of the particles passing through the two slits were collected by the EMFC. Each slit was driven by stepping motors. The data acquisition system of the emittance monitors was constructed using a Windows PC. The stepping motors were controlled using an Interface [15] CSI-742020 motor controller. The currents from the EMFCs were terminated with a $1-\mathrm{k} \Omega$ terminator, amplified by a factor of 11 and converted to a voltage signal by an operational amplifier. The voltage signal was digitized with a CAEN [16] digitizer DT5720 and read out by the $\mathrm{PC}$ via a USB connection. The default sampling frequency of the DT5720 is $250 \mathrm{MHz}$, but to reduce the data volume, the sampling frequency was set to $30 \mathrm{MHz}$. The data acquisition and analysis programs were written in $\mathrm{C}++$ language.

Finally, the beam was directed to the beam dump. The beam dump consists of a copper cylinder cut with an angle of 30 degrees and a 1-mm tungsten plate attached to the cut surface of the cylinder by the HIP method. Because contamination by impurities such as nickel and copper cause neutron radiation, a high purity tungsten was used for the beam target.

The emittance monitor chamber was evacuated by a 400-L/s ion pump. Typical internal pressures of the test bench were $1.0 \times 10^{-5}$ Pa without the beam and $1.8 \times 10^{-5}$ Pa with the $50-\mathrm{mA}$ beam.

\section{RFQ III CONDITIONING RESULTS}

Before starting the beam experiments, RFQ III was conditioned at the RFQ test stand.

After all the components were assembled, the measured unloaded $Q$ value was 10100 [7]. This corresponds to $90 \%$ of a SUPERFISH [17] calculation. The field uniformity of the quadrupole modes was $1 \%$, and the dipole-mode mixing rate was less than $2 \%$. The coupling factor of the rf coupler was set to 1.9 .

Figure 4 shows the conditioning history of RFQ III. RFQ III was conditioned up to $25 \mathrm{~Hz}$ because the available klystron at the RFQ test stand at that time was capable of operation up to only $25 \mathrm{~Hz}$. First, the pulse width and repetition rate were set to $30 \mu \mathrm{s}$ and $25 \mathrm{~Hz}$, respectively. After $10 \mathrm{~h}$ of conditioning, the peak power $P_{\text {peak }}$ reached $460 \mathrm{~kW}$, which is $15 \%$ higher than the nominal peak power of $400 \mathrm{~kW}$. The pulse width was gradually broadened to $600 \mu$ s. In Fig. 4, the solid (black) line represents $P_{\text {peak }}$, and the dashed line (blue) represents the averaged power $P_{a v}$.

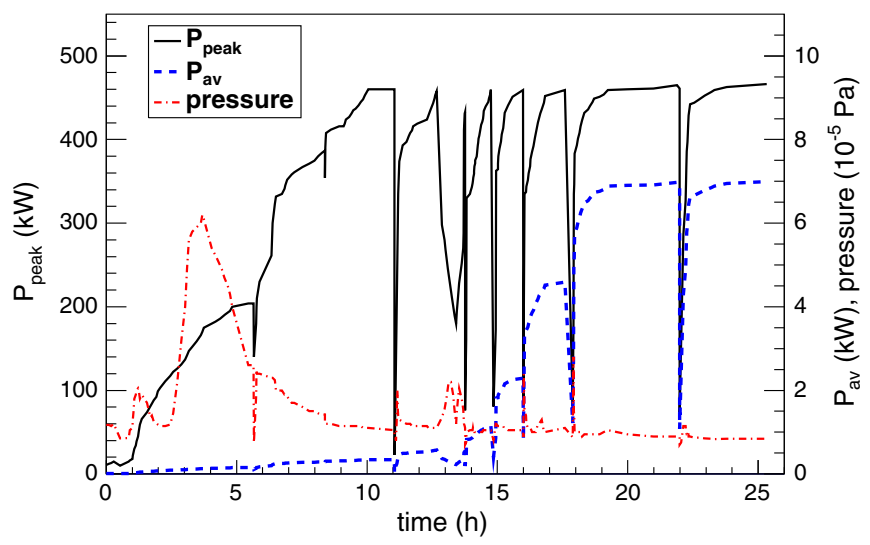

FIG. 4. Conditioning history of RFQ III.
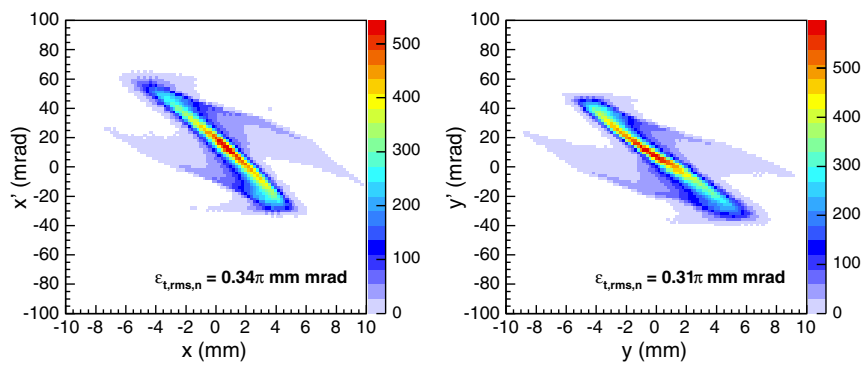

FIG. 5. Phase space distribution of the ion source measured just after the SM1 of the ion source test stand. 


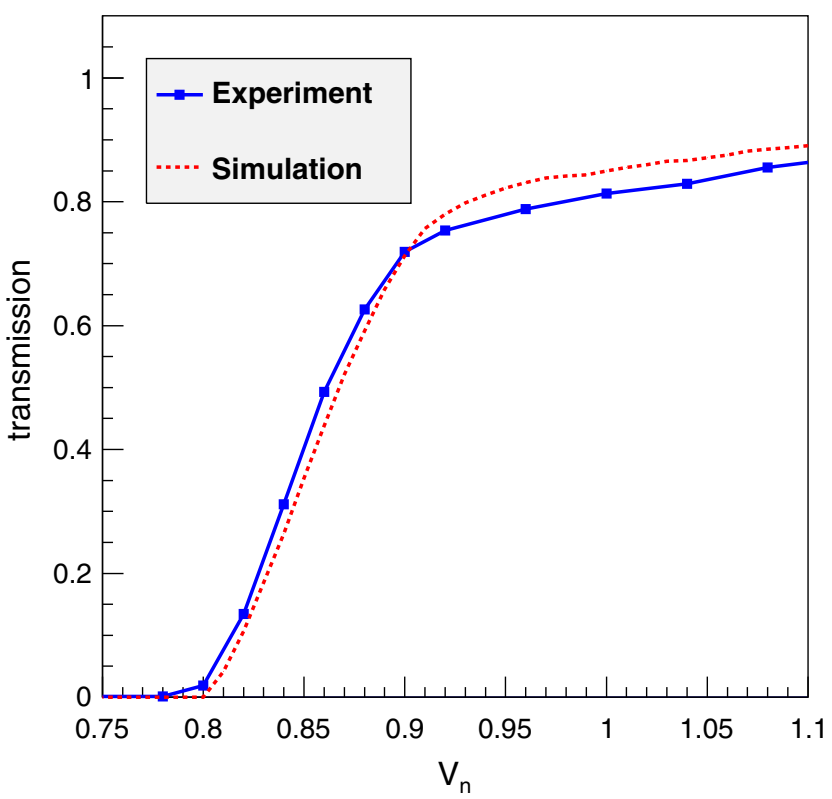

FIG. 6. Measured and simulated transmissions of RFQ III as functions of the intervane voltage $V_{n}$.

The dash-dotted (red) line represents the vacuum pressure in the cavity.

After that, to reduce the reflection rate of the $\mathrm{rf}$ at the $600 \mu \mathrm{s}$ and $25 \mathrm{~Hz}$ operation, the vane-cooling water temperatures were set to $26.8^{\circ} \mathrm{C}$, and the wall-cooling water temperatures were set to $27.0^{\circ} \mathrm{C}$.

\section{BEAM TEST RESULTS}

In this section, the results of the $50-\mathrm{mA} \mathrm{H}^{-}$beam experiment are presented and compared with those of
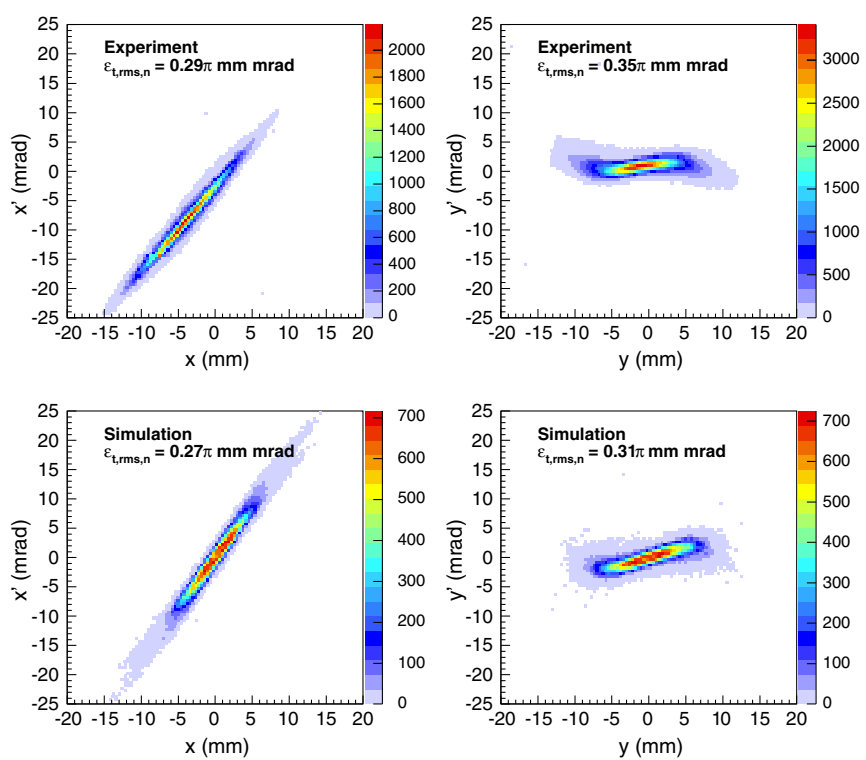

FIG. 7. Measured (upper row) and simulated (lower row) transverse emittances of RFQ III. The absolute positions of the emittance monitors are not fully calibrated.

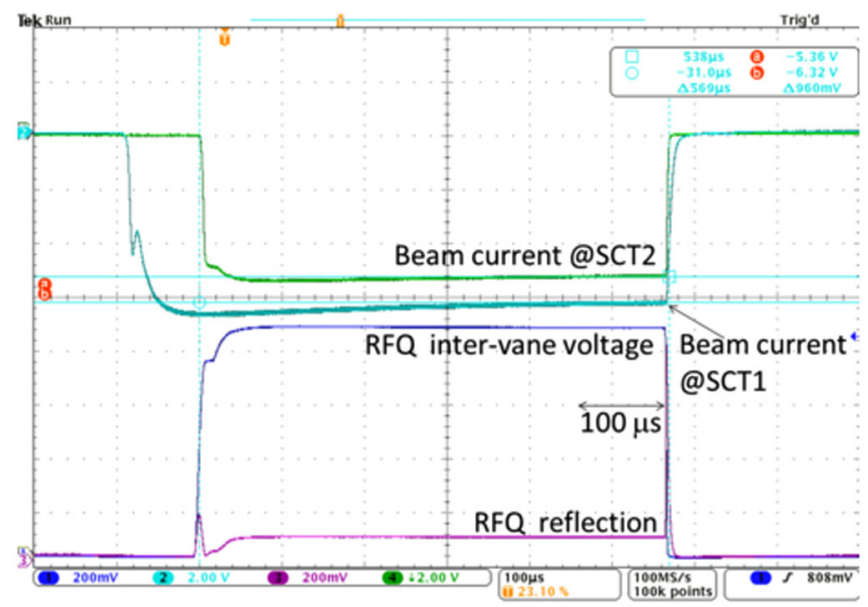

FIG. 8. Typical wave form of the $500 \mu$ s pulse operation. Repetition rate of this operation was $5 \mathrm{~Hz}$.

the simulation. LINACSRFQSIM was employed for the RFQ simulation, and PARMILA [18] was employed for the test bench simulation. For the input distribution into the $\mathrm{RFQ}$, the generated distribution based on the measurement at the LEBT was used. Figure 5 shows the measured phase space distribution at the LEBT.

Because the LEBT of the RFQ test stand was not equipped with emittance monitors, the distribution of the ion source had been measured at another ion source test stand prior to installation on the RFQ test stand. It is assumed that there is no significant difference between the effects of the space

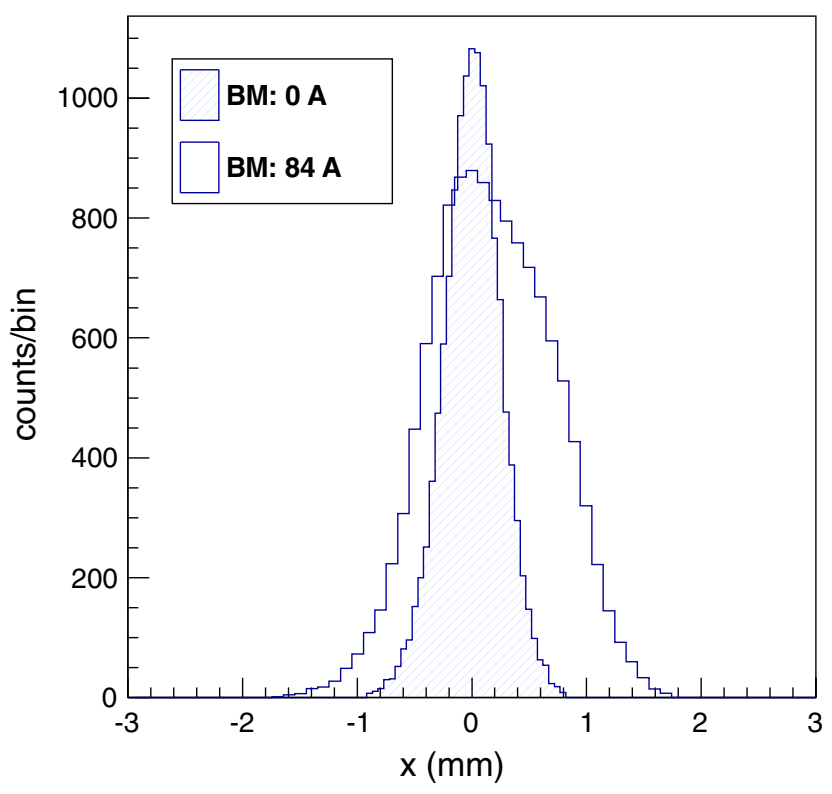

FIG. 9. Profiles of the beams having passed through the slits (SL) and bending magnet (BM). The solid histogram is the profile when the $\mathrm{BM}$ is set to $0 \mathrm{~A}$ (straight beam), and the hatched histogram is that when the BM is set to $84 \mathrm{~A}$ (bended by 15 degrees). The peak positions of each histogram are adjusted to overlay. 

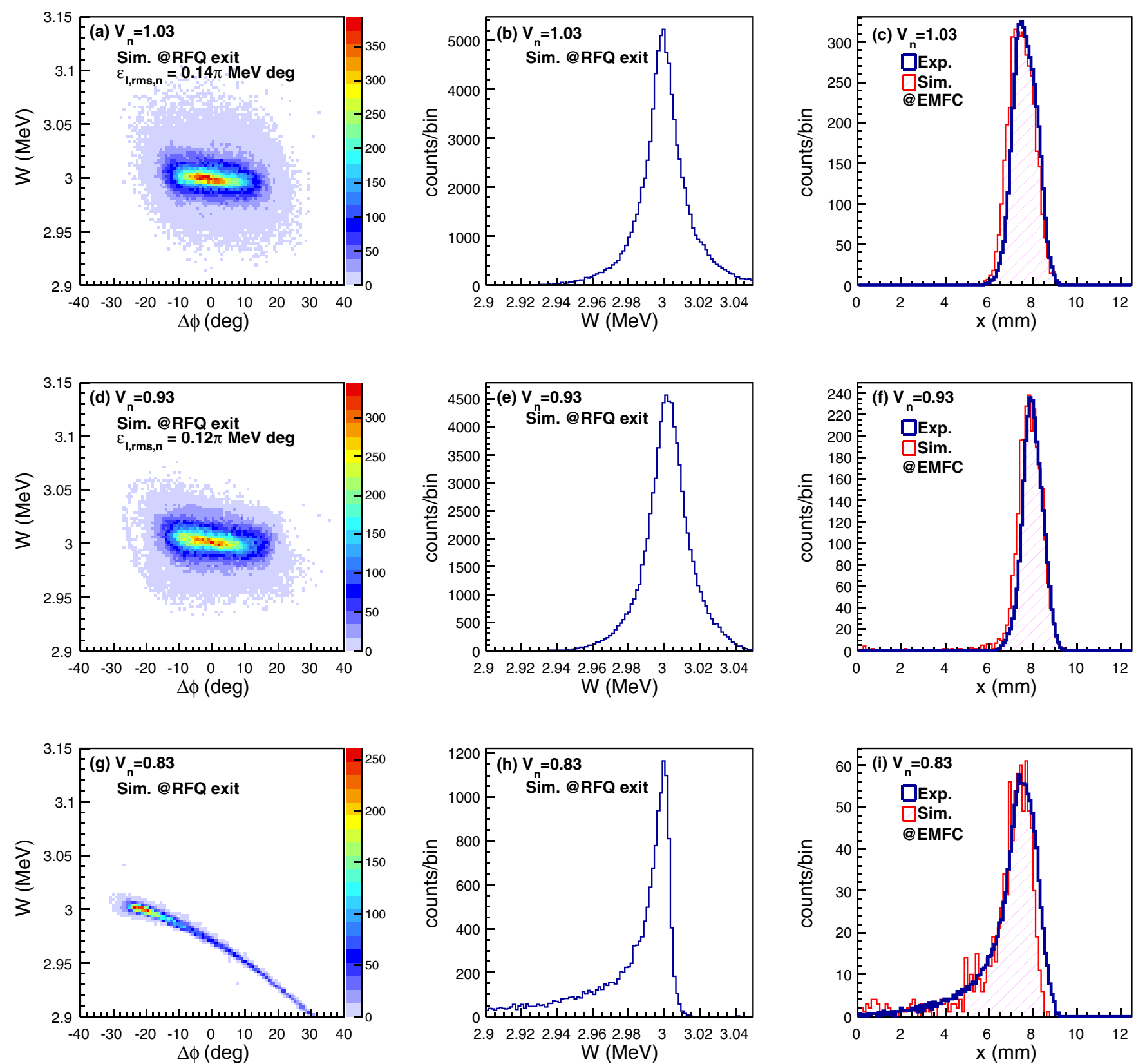

FIG. 10. Longitudinal emittances and energy spreads at the exit of RFQ III, where the simulation results are given in (a), (b), (d), (e), $(\mathrm{g})$, and $(\mathrm{h})$, and the measured and simulated transverse beam profiles at the EMSLx are given in (c), (f), and (i) for BM = 84 A. Exp. and Sim. denote the experiment and simulation results, respectively.

charge neutralization of the two test stands. The input distribution for LINACSRFQSIM was reconstructed from the measured distribution using GENBEAM [19].

Figure 6 shows the intervane voltage dependence of the transmission through the RFQ.

$V_{n}$ denotes the intervane voltage of the RFQ normalized to the nominal voltage, and the vertical axis is the transmission. The solid and dashed lines represent the measured and simulated transmissions of the accelerated particles, respectively. The accelerated beam current was obtained by measuring the 324-MHz spectrum of the FCT2 signal using a Tektronix [20] MDO4014B-3 mixed-domain oscilloscope.
The measured beam current was normalized by the RFQ injection current measured with the SCT1 of the LEBT. The measured and simulated transmissions agree with each other within $4 \%$.

Next, the results of the transverse emittance measurements of the 50-mA beam are shown in Fig. 7.

To prevent melting of the edge of the slits, the emittances of the $50-\mathrm{mA}$ beam were measured with a width of $100 \mu \mathrm{s}$ and a repetition rate of $5 \mathrm{~Hz}$. Figure 8 shows a typical wave form of the beam with a $500 \mu$ s pulse length.

In this figure, the current at SCT1 is the ion source current and the current at SCT2 is that at the RFQ exit. 


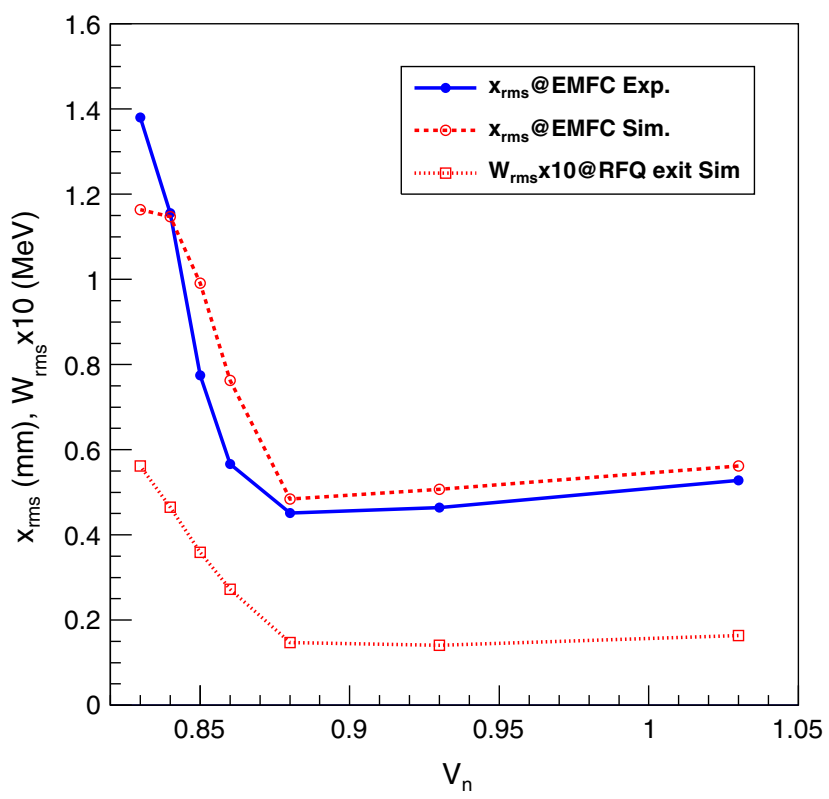

FIG. 11. Measured and simulated beam widths at the EMFCx as functions of $V_{n}$. The simulated energy spreads at the RFQ exit are also shown.

There is no significant change of the transmission during this $500 \mu$ s pulse, therefore, it is believed that the space charge neutralization is sufficiently high at the beginning of the $500 \mu$ s beam pulse. The last $100 \mu \mathrm{s}$ was used for the emittance measurement. To extract this $100 \mu \mathrm{s}$, the timing and pulse length of the ion source was fixed and the timing of the RFQ was shifted. Figure 5 was measured with similar timing.

In Fig. 7, the upper row shows the measured transverse emittances and the lower row gives the simulation results. The measured values of the normalized rms transverse emittances $\varepsilon_{t, r m s, n}$ in the horizontal and vertical planes were $0.29 \pi \mathrm{mm} \mathrm{mrad}$ and $0.35 \pi \mathrm{mm} \mathrm{mrad}$, whereas the simulated emittances were $0.27 \pi \mathrm{mmmrad}$ and $0.31 \pi \mathrm{mmmrad}$, respectively. The simulation well reproduces the measurements, indicating that the transverse beam performance of RFQ III conforms to its design.

Finally, the results of the energy spread measurement of the $50-\mathrm{mA}$ beam are presented. The energy spread was estimated by measuring the beam profile bended by the $\mathrm{BM}$ and then comparing it with the simulation. To reduce the transverse emittance effect from the beam profile, the SL was inserted just upstream of the BM. In Fig. 9, the measured profiles of the beams having passed through the SL and the BM with BM settings of 0 and $84 \mathrm{~A}$ are shown. These profiles were measured with the EMFCx.

Then, with a BM setting of $84 \mathrm{~A}$, beam profiles were measured by varying $V_{n}$ and compared with the simulation. Figure 10 represents the simulation results of the longitudinal emittances (first column) and energy spread (second column) at the RFQ exit, and the measured and simulated beam profiles at the EMSLx (third column) for three values of $V_{n}$, where each row represents the case of $V_{n}=1.03$,
0.93 , and 0.83 . The $\mathrm{W}$ and $\phi$ denote the energy and phase of the particles. The simulated normalized longitudinal rms emittance $\varepsilon_{l, r m s, n}$ and energy spread at the exit of the RFQ for $V_{n}=1.03$ are $0.14 \pi \mathrm{MeV}$ deg and $16 \mathrm{keV}$, respectively.

The longitudinal emittance of $V_{n}=0.93$ (d) is broadened in the phase direction and reduced in the energy direction as compared with that of $V_{n}=1.03$ (a). This behavior is indicative of a reduced energy spread, which can also be seen in a comparison between the cases (b) and (e). Because the energy spread is smaller, the beam profile measured with the EMSLx (f) is reduced compared with that shown in (c). In the figure (g), showing the longitudinal emittance of $V_{n}=0.83$, the particles are spilling away from the separatrix. Therefore, the energy distribution (h) and the beam profile (i) have long tails toward the lowenergy sides. The excellent agreement between the measured and simulated beam profiles indicates that the energy spread is well measured by this method.

In Fig. 11, the measured and simulated beam widths at the EMFCx and the simulated energy spread at the exit of the RFQ for each $V_{n}$ are shown.

The horizontal axis represents $V_{n}$. The term $x_{r m s}$ denotes the rms value of the beam profile, and $W_{r m s}$ is the simulated rms energy spread. The measured and simulated values of $x_{r m s}$ as a function of $V_{n}$ agree well with each other.

From these results, the longitudinal performance of RFQ III also conforms to its design.

\section{DISCUSSION}

The measured transmission of RFQ III at the nominal intervane voltage is $81 \%$, which is lower than the design value [3]. This is because the actual transverse emittance of the ion source (in Fig. 5) is larger than that assumed in the design of RFQ III, which is $0.20 \pi \mathrm{mm}$ mrad (normalized, rms). Additionally, Fig. 5 shows significant filamentations, and this is quite different from the assumed water-bag distribution. With the present beam current of the ion source and the transmission of the RFQ, the required current of $50 \mathrm{~mA}$ at the linac exit can be achieved, but the transmission can be improved to $86 \%$ by increasing the intervane voltage of the RFQ to $110 \%$, if necessary. Of course, higher transmission is expected if higher voltage is applied, however, this may require more conditioning than that of the present level (15\% higher than the nominal voltage). Currently, we consider that such operation is not necessary. The emittances at the RFQ exit is also higher than expected, but they are still acceptable for the following linac. The effort to reduce the ion source emittance should improve the transmission and emittance of the RFQ.

\section{CONCLUSION}

A beam test of J-PARC RFQ III was successfully conducted. RFQ III employs a conventional beam dynamics 
design, namely, it consists of an RMS, a SP, a GB, and an ACC. Except for the injection section, the intervane voltage is maintained constant. The equipartitioning condition was adopted in the GB, which is the most critical section.

After $20 \mathrm{~h}$ of conditioning, the peak power reached $460 \mathrm{~kW}$, which corresponds to $115 \%$ of the nominal power of $400 \mathrm{~kW}$, with a pulse width of $600 \mu \mathrm{s}$ and a repetition rate of $25 \mathrm{~Hz}$.

Before installing RFQ III into the accelerator tunnel, an off-line beam test using a $\mathrm{H}^{-}$beam was performed and the experimental data were compared with the simulation results. LINACSRFQSIM was used for the simulation of the RFQ, and PARMILA was used for simulation of the test bench. A distribution based on the measured distribution at the LEBT was used for the input distribution of the RFQ simulation.

As for the transmission of the 50-mA beam, the measurement and the simulation agreed well within $4 \%$. The horizontal and vertical emittances were measured to be $0.29 \pi \mathrm{mm} \mathrm{mrad}$ and $0.35 \pi \mathrm{mm} \mathrm{mrad}$, whereas the simulation results were $0.27 \pi \mathrm{mm}$ mrad and $0.31 \pi \mathrm{mm} \mathrm{mrad}$, respectively. The simulation reproduced the experimental results quite well. Moreover, the longitudinal emittance and the energy spread at the RFQ exit obtained from the simulation, given as $0.14 \pi \mathrm{MeV}$ deg and $16 \mathrm{keV}$, respectively, were confirmed using the energy-analyzing BM. Using the $\mathrm{BM}$ at a setting of $84 \mathrm{~A}$ induced a 15-degree bend in the beam, and the beam after the BM was measured and compared with that of the simulation. The measured and simulated profiles showed excellent agreement.

From the results described above, it is concluded that the performance of J-PARC RFQ III conforms to its design.

\section{ACKNOWLEDGMENTS}

The authors would like to thank Professor Robert A. Jameson for fruitful discussions about the particle simulations of the RFQ. We express their appreciation to the many manufacturing companies involved in this project. In particular, TOYAMA Co., Ltd., Nakaya seisakusyo Co. Ltd., and Metal Technology Co. Ltd., contributed remarkably to the fabrication of RFQ III.
[1] T. Koseki and K. Hasegawa, in Proceedings of the 5th International Particle Accelerator Conference, IPAC-2014, Dresden, Germany, 2014 (JACoW, Dresden, Germany, 2014), pp. 3373-3375.

[2] A. Ueno and Y. Kondo in Proceedings of the 20th International Linac Conference, LINAC-2000, Monterey, CA, 2000 (SLAC, Menlo Park, CA, 2000), pp. 545-547.

[3] Y. Kondo, K. Hasegawa, T. Morishita, and R. A. Jameson, Phys. Rev. ST Accel. Beams 15, 080101 (2012).

[4] R. A. Jameson, Oak Ridge National Laboratory Technical Report No. ORNL/TM-2007/001, 2007.

[5] R. A. Jameson, Technical Report No. KEK/J-PARC, 2012.

[6] R. A. Jameson, IEEE Trans. Nucl. Sci. 28, 2408 (1981).

[7] T. Morishita, Y. Kondo, H. Oguri, K. Hasegawa, H. Kawamata, T. Sugimura, and F. Naito, in Proceedings of the 4th International Particle Accelerator Conference, IPAC-2013, Shanghai, China, 2013 (JACoW, Shanghai, China, 2013), pp. 3839-3841.

[8] K. Hasegawa, T. Kobayashi, Y. Kondo, T. Morishita, H. Oguri, Y. Hori, C. Kubota, H. Matsumoto, F. Naito, and M. Yoshioka, in Proceedings of the International Particle Accelerator Conference, Kyoto, Japan (ICR, Kyoto, 2010), pp. 621-623.

[9] Y. Kondo, T. Morishita, K. Hasegawa, E. Chishiro, K. Hirano, T. Hori, H. Oguri, F. Sato, S. Shinozaki, T. Sugimura, H. Kawamata, F. Naito, Y. Fukui, K. Futatsukawa, and K. Nanmo, Phys. Rev. ST Accel. Beams 16, 040102 (2013).

[10] A. Ueno, I. Koizumi, K. Ohkoshi, K. Ikegami, A. Takagi, S. Yamazaki, and H. Oguri, Rev. Sci. Instrum. 85, 02B133 (2014).

[11] Thamway Co. Ltd., http://www.thamway.co.jp/.

[12] Mitsubishi Materials Co., http://www.mmc.co.jp/.

[13] H. Matsumoto, M. Akemoto, H. Hayano, A. Miura, T. Naito, and S. Takeda, in Proceedings of the 1991 Particle Accelerator Conference, San Francisco, CA, 1991 (IEEE, New York, 1991), pp. 1008-1010.

[14] Toshiba Electron Tubes and Devices Co. Ltd., http://www .toshiba-tetd.co.jp/.

[15] Interface Co., http://www.interface.co.jp/.

[16] CAEN, http://www.caen.it/csite/.

[17] J. H. Billen and L. M. Young, Technical Report No. LA-UR96-1834, 1996

[18] H. Takeda, Technical Report No. LA-UR-98-4478, 1998.

[19] D. Bouma and J. Stovall, Technical Report, 2009.

[20] Tektronix Inc., http://www.tek.com/. 